Chronic clozapine treatment improves prenatal infectioninduced working memory deficits without influencing adult hippocampal neurogenesis

\author{
Journal Article \\ Author(s): \\ Meyer, Urs; Knuesel, Irene; Nyffeler, Myriel; Feldon, Joram \\ Publication date: \\ 2010-03 \\ Permanent link: \\ https://doi.org/10.3929/ethz-b-000015960
}

Rights / license:

In Copyright - Non-Commercial Use Permitted

Originally published in:

Psychopharmacology 208(4), https://doi.org/10.1007/s00213-009-1754-6 


\title{
Chronic clozapine treatment improves prenatal infection-induced working memory deficits without influencing adult hippocampal neurogenesis
}

\author{
Urs Meyer • Irene Knuesel • Myriel Nyffeler • \\ Joram Feldon
}

Received: 11 September 2009 /Accepted: 3 December 2009/Published online: 30 December 2009

(C) Springer-Verlag 2009

\begin{abstract}
Background Converging evidence indicates that prenatal exposure to immune challenge can induce long-term cognitive deficits relevant to schizophrenia. Such cognitive impairments may be related to deficient hippocampal neurogenesis at adult age.

Objectives In the present study, we sought evidence for the possibility that chronic treatment with the reference atypical antipsychotic drug clozapine may improve prenatal infection-induced cognitive dysfunctions by stimulating adult hippocampal neurogenesis.

Methods This hypothesis was tested in a well-established mouse model of prenatal immune challenge which is based on prenatal administration of the viral mimic, polyriboinosinicpolyribocytidilic acid (PolyI:C).

Results We found that maternal PolyI:C (5 mg/kg, i.v.) exposure on gestation day 17 led to significant spatial working memory impairment and reduced hippocampal neurogenesis in the resulting offspring at adult age. The latter effect was apparent in postmortem immunohistochemical analyses of the cell proliferation marker bromodeoxyuridine and the microtubule-associated protein doublecortin, a marker of newborn neuronal cells. Chronic (3 weeks) administration of clozapine ( $5 \mathrm{mg} / \mathrm{kg} / \mathrm{day}$, i.p.) significantly improved the prenatal PolyI:C-induced work-
\end{abstract}

\footnotetext{
U. Meyer $(\bowtie) \cdot$ I. Knuesel $\cdot$ M. Nyffeler $\cdot$ J. Feldon

Laboratory of Behavioural Neurobiology,

Swiss Federal Institute of Technology (ETH) Zurich,

Schorenstrasse 16,

8603 Schwerzenbach, Switzerland

e-mail: urmeyer@ethz.ch

I. Knuesel

Institute of Pharmacology and Toxicology, University of Zurich,

Winterthurerstrasse 190,

8057 Zurich, Switzerland
}

ing memory deficits, while at the same time, it negatively affected working memory performance in adult offspring born to control mothers. These bidirectional cognitive effects of clozapine were not paralleled by concomitant effects on adult hippocampal neurogenesis.

Conclusions Our findings do not support the hypothesis that the atypical antipsychotic drug clozapine may influence cognitive functions by acting on adult neurogenesis in the hippocampus, regardless of whether the drug is administered to subjects with or without a neurodevelopmental predisposition to adult neuropathology.

Keywords Animal model - Antipsychotic drugs . Cognition · Hippocampus $\cdot$ Infection $\cdot$ Schizophrenia

\section{Introduction}

Maternal infection during pregnancy is a known environmental risk factor for the development of schizophrenia and related disorders (Brown and Susser 2002; Fatemi 2005; Brown 2006; Patterson 2007). Even though the precise neuroimmunological mechanisms involved still need to be delineated, one prevalent hypothesis suggests that infectioninduced disruption of fetal neurodevelopmental processes may predispose the organisms to long-lasting changes in subsequent brain and behavioral development, thereby increasing the risk of psychotic disturbances in later life (Gilmore and Jarskog 1997; Patterson 2002; Fatemi 2005; Meyer et al. 2007, 2009b). This hypothesis has been substantiated by numerous investigations in experimental rodent models demonstrating the emergence of altered fetal brain development (Meyer et al. 2008a) and multiple longterm brain and behavioral abnormalities relevant to schizophrenia following prenatal exposure to infection and/or 
immune activation (for recent reviews, see Meyer et al. 2009a; Meyer and Feldon 2009; Patterson 2009).

One of the critical psychopathological outcomes of prenatal immune challenge is the appearance of cognitive impairments. Experimental data show that prenatal exposure to immune-activating agents such as the bacterial endotoxin lipopolysaccharide (LPS), the viral mimic polyriboinosinic-polyribocytidilic acid (PolyI:C), or the pro-inflammatory cytokine interleukin-6 induces long-term cognitive impairments in the form of deficiencies in spatial working memory (Meyer et al. 2005, 2008b) and reference memory (Samuelsson et al. 2006), novel object recognition (Ozawa et al. 2006; Coyle et al. 2009), and discrimination reversal learning (Zuckerman and Weiner 2005; Meyer et al. 2006). These experimental findings are complemented by the recent pioneering findings by Brown and colleagues showing that prenatal exposure to viral infection is associated with executive dysfunctions in patients with schizophrenia (Brown et al. 2009). Hence, there is converging evidence derived from both experimental and epidemiological studies supporting a causal relationship between prenatal immune challenge and emergence of cognitive impairments relevant to schizophrenia.

It has recently been demonstrated that impaired adult neurogenesis in the hippocampus (HPC) is involved in the cellular neuropathology of schizophrenia (Reif et al. 2006). Adult neurogenesis refers to the generation of new neurons in the adult brain. This complex multistep process includes phases of proliferation of progenitor cells, followed by differentiation and maturation into a neuronal (or glial) phenotype (for a recent review, see Balu and Lucki 2009). The HPC is one of the major neurogenic brain regions in which neurogenesis can occur throughout life (Altman and Das 1965; van Praag et al. 2002; Kempermann et al. 2004). This brain structure is also known to be critically involved in fundamental cognitive functions, especially in spatial memory (Olton and Papas 1979; Moser et al. 1993; Lee and Kesner 2003; Bannerman et al. 2004). Furthermore, structural and functional abnormalities in the HPC are believed to critically mediate at least some of the cognitive abnormalities in patients with schizophrenia (Harrison 1999, 2004; Weinberger 1999; Antonova et al. 2004; Tamminga 2006). Based on the recent findings by Reif and colleagues (2006), it has been proposed that impaired adult neurogenesis in the HPC may provide an important (but relatively unexplored) cellular link between hippocampal abnormalities and emergence of cognitive dysfunctions in patients with schizophrenia (Reif et al. 2007; Toro and Deakin 2007; Kempermann et al. 2008). Furthermore, it has been hypothesized that the beneficial effects of atypical antipsychotic drugs on cognitive abnormalities in schizophrenic individuals may be accounted for, at least in part, by an amelioration of deficient hippocampal neurogenesis (Reif et al. 2006, 2007; Toro and Deakin 2007).

In keeping with the role of prenatal infection in the etiology of schizophrenia in general and with its impact on adult cognitive functions in particular, prenatal immune challenge may induce cognitive disturbances by disrupting postnatal neurogenesis. Initial support for this hypothesis has been obtained by recent experimental studies in mice and rats demonstrating that offspring born to gestationally immune-challenged mothers display reduced postnatal hippocampal neurogenesis (Meyer et al. 2006; Cardon et al. 2009; Cui et al. 2009) together with marked deficits in various cognitive tasks (reviewed in Meyer et al. 2009a, b; Meyer and Feldon 2009). However, despite evidence for beneficial effects of atypical antipsychotic drugs on cognitive impairments induced by prenatal immune challenge (Ozawa et al. 2006), it remains essentially unknown whether these procognitive effects may be related to possible effects of atypical antipsychotic drugs on adult hippocampal neurogenesis.

Therefore, the present study was designed to test the hypothesis that chronic treatment with the reference atypical antipsychotic drug clozapine (CLZ) may improve prenatal infection-induced cognitive dysfunctions by stimulating adult hippocampal neurogenesis. For this purpose, we used a well-established experimental mouse model of prenatal exposure to a viral-like acute phase response, which is based on maternal administration of the synthetic analog of double-stranded RNA, PolyI:C (Meyer et al. 2009a). The effects of prenatal immune challenge on adult cognitive functions were assessed using a spatial working memory paradigm known to be sensitive to detect the longterm cognitive effects of prenatal PolyI:C exposure in mice (Meyer et al. 2005, 2008b). Adult hippocampal neurogenesis was first evaluated by immunohistochemical analyses of bromodeoxyuridine (BrdU) in order to label mitotically active cells (Dolbeare 1995). In addition, we performed immunohistochemical investigations of doublecortin (DCX), a microtubule-associated protein expressed specifically in newborn neuronal but not glial cells (Francis et al. 1999; Brown et al. 2003; Couillard-Despres et al. 2005). The latter investigations were conducted in order to confirm the neuronal phenotype of newly born cells in the adult HPC.

\section{Materials and methods}

Animals

C57BL6/J mice were used throughout the study. Female and male breeders were obtained from our in-house specific pathogen-free colony at the age of 10-14 weeks. Breeding 
began after 2 weeks of acclimatization to the new animal holding room, which was a temperature- and humiditycontrolled $\left(21 \pm 1^{\circ} \mathrm{C}, 55 \pm 5 \%\right)$ holding facility under a reversed light-dark cycle (lights off 0800-2000 hours). All animals had ad libitum access to standard food (Kliba 3430, Klibamühlen, Kaiseraugst, Switzerland) and water. All procedures described in the present study had been previously approved by the Cantonal Veterinary Office in Zurich and were in agreement with the Principles of Laboratory Animal Care (NIH publication no. 86-23, revised 1985). All efforts were made to minimize distress and number of animals used.

Prenatal immune activation

For the purpose of the prenatal immunological manipulation, female mice were subjected to a timed mating procedure as described previously (Meyer et al. 2005). Pregnant dams on gestation day (GD) 17 received either a single injection of PolyI:C (potassium salt; Sigma-Aldrich, Buchs, St. Gallen, Switzerland) or control (CON; saline) treatment. A total of ten pregnant mice were included, half of which were subjected to PolyI:C treatment and the other half to saline treatment. PolyI:C (5 mg/kg) was dissolved in sterile and pyrogen-free isotonic $0.9 \% \mathrm{NaCl}$ (saline) solution to yield a final concentration of $1 \mathrm{mg} / \mathrm{ml}$. All solutions were administered via the intravenous (i.v.) route at the tail vein under mild physical constraint with an injection volume of $5 \mathrm{ml} / \mathrm{kg}$. All animals were returned to their home cages immediately after the injection procedure and left undisturbed until weaning of the offspring.

The dose of PolyI:C (i.e., $5 \mathrm{mg} / \mathrm{kg}$ ) was selected based on our previous studies (e.g., Meyer et al. 2005, 2006, 2008b). The chosen gestational window (i.e., GD 17) corresponds roughly to the middle-to-late second trimester of human pregnancy in terms of developmental biology and percentage of gestation (Clancy et al. 2001, 2007). The selection of this gestational stage was based on our previous investigations in mice, which demonstrate that prenatal immune challenge especially in late gestation leads to marked spatial working memory deficits at adult age (Meyer et al. 2008b).

\section{Chronic CLZ treatment and BrdU injections}

Offspring born to PolyI:C- or control-treated mothers were weaned and sexed at postnatal day (PND) 21 . Littermates of each sex were caged separately and kept in groups of four to six animals per cage. All animals were maintained under ad libitum food and water diet and kept in a temperature- and humidity-controlled animal vivarium under a 12:12-h reversed light-dark cycle as described above.
The chronic drug regime started when the offspring reached 12 weeks of age and lasted for 3 weeks (see schematic representation of the study design in Fig. 1a). During this period, the animals were given daily injections of CLZ $(5 \mathrm{mg} / \mathrm{kg}$ ) or vehicle (VEH) via the intraperitoneal (i.p.) route. The dose of CLZ was based on previous studies showing that chronic ( 3 weeks) CLZ exposure at a dose of $5 \mathrm{mg} / \mathrm{kg}$ (i.p.) is efficient in improving prenatal PolyI:Cinduced cognitive dysfunctions in adult mice (see Ozawa et al. 2006). CLZ powder (Novartis, Basel, Switzerland) was first dissolved in $0.1 \mathrm{~N}$ hydrochloric acid $(\mathrm{HCl})$ in $0.9 \%$ isotonic $\mathrm{NaCl}$ solution and then neutralized with $\mathrm{Na}_{2} \mathrm{CO}_{3}$ to yield a final $\mathrm{pH}$ of $~ 5.5$. VEH-treated animals received $\mathrm{NaCl}$ solution with the appropriate amounts of $0.1 \mathrm{~N} \mathrm{HCl}$ and $\mathrm{Na}_{2} \mathrm{CO}_{3}(\mathrm{pH} \sim 5.5)$.

One day following cessation of the chronic CLZ or VEH treatment, all animals were assigned to a BrdU injection regime following a modified protocol described by Kralic et al. (2005). BrdU (Sigma, Switzerland) was dissolved in $0.9 \% \mathrm{NaCl}$ and administered i.p. twice daily (0900 and 1900 hours) on three subsequent days at a concentration of $50 \mathrm{mg} / \mathrm{kg}$ in a $10-\mathrm{mg} / \mathrm{ml}$ volume (see Fig. 1a).

Each experimental group consisted of one to two offspring derived from multiple independent litters (five PolyI:C litters and five control litters). Only male subjects were included in the working memory test and subsequent postmortem immunohistochemical investigations. The number of subjects in each of the four experimental groups (i.e., CLZ-treated PolyI:C offspring, VEH-treated PolyI:C offspring, CLZ-treated CON offspring, and VEH-treated CON offspring) was $N=8$ male offspring.

\section{Cognitive phenotyping}

Cognitive testing of CLZ- or VEH-treated PolyI:C and control offspring was performed using a spatial working memory task in the Morris water maze. The test of spatial working memory in the Morris water maze commenced 1 day after the third BrdU injection day (see Fig. 1a). The apparatus consisted of a white fiberglass tank (1 $\mathrm{m}$ diameter) as fully described before (Meyer et al. 2005). It was positioned in the middle of a well-lit room enriched with distal spatial cues and contained a clear Plexiglas cylinder ( $7 \mathrm{~cm}$ in diameter) to provide an escape platform.

After a 1-day pretraining session with the platform visible in the center of the maze, the animals were tested in a working memory task lasting for eight consecutive days. The working memory test was based on the matching-toposition paradigm, in which the animals were required to learn the novel position of a hidden platform located on trial one of each day in order to navigate effectively to the same location (i.e., matching) on the subsequent trial on the 

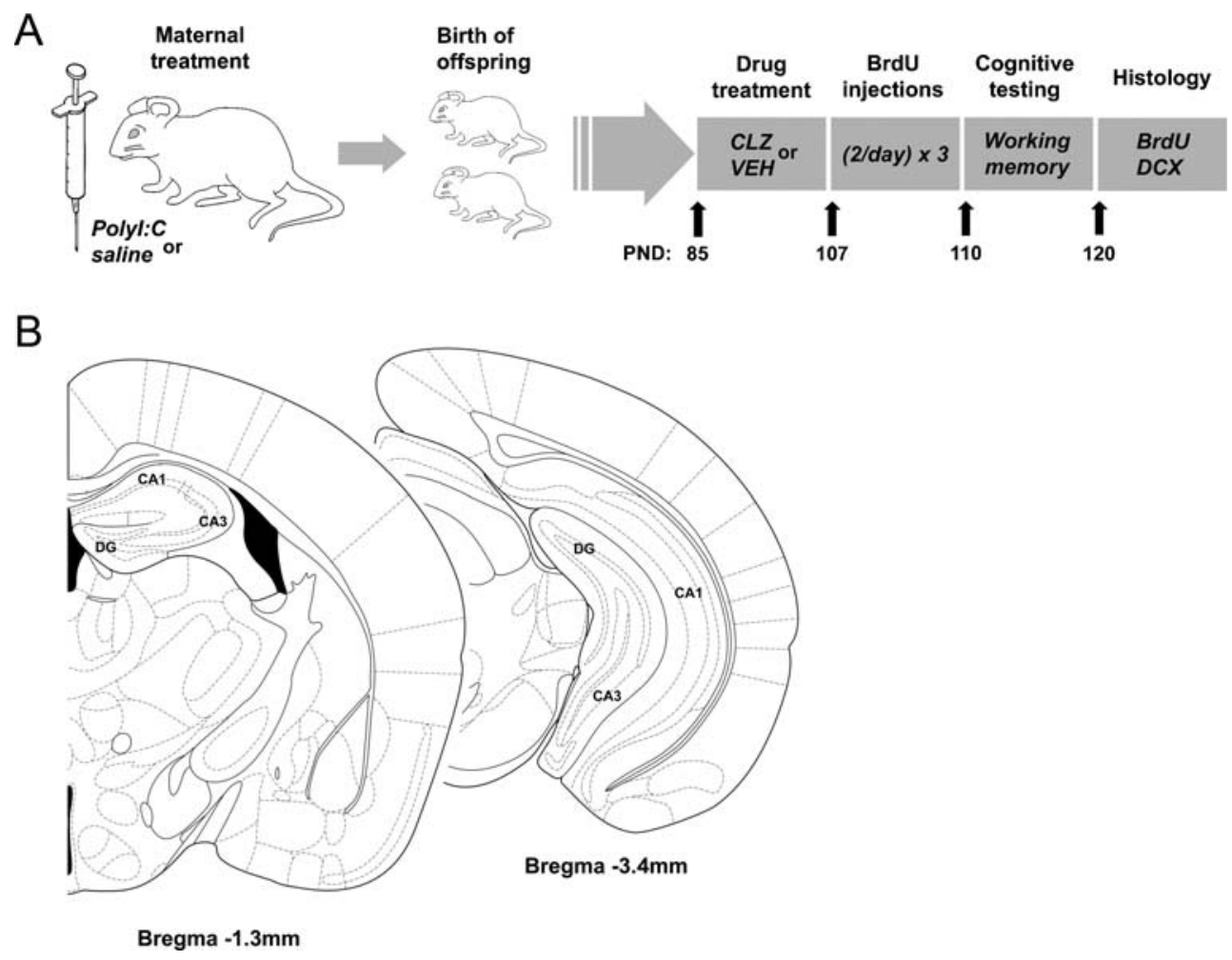

Fig. 1 a General study design. Pregnant C57BL/6 mice were treated with PolyI:C (5 mg/kg, i.v.) or corresponding control (saline) solution on gestation day 17. The resulting offspring were then subjected to chronic clozapine ( $C L Z ; 5 \mathrm{mg} / \mathrm{kg} /$ day, i.p.) or vehicle $(V E H)$ treatment when they reached the adult stage of development, i.e., for 3 weeks starting from PND 85-106. One day following cessation of the chronic drug regime, all offspring were injected with bromodeoxyuridine $(B r d U ; 50 \mathrm{mg} / \mathrm{kg})$ twice daily on three subsequent days (PND 107-109). The offspring were then subjected to cognitive testing in a spatial working memory task on the next 8 days (PND 100-118). Two days after completion of cognitive testing (PND 120), all animals were

same day (Steele and Morris 1999). Hence, each test day included two trials. The hidden platform remained in the same position across trials on a given test day but took a new position on each test day. The daily change of the platform position ensured that the information acquired on a given day was irrelevant for the next day, thus taxing the flexible use of working memory (Baddeley 2003). To begin each trial, an animal was placed gently in the water maze against the maze wall at one of the four cardinal positions $(\mathrm{N}, \mathrm{E}, \mathrm{S}, \mathrm{W})$ and was allowed a maximum of $60 \mathrm{~s}$ to locate the platform. If it failed to reach the platform, it was then guided to it by the experimenter. Each animal was allowed to spend $10 \mathrm{~s}$ on the platform before commencement of the next trial.

To manipulate the retention demand in the temporal domain, the intertrial interval varied between trials 1 and 2 . First, a "minimal delay" of 10-15 s was used for four consecutive days (days 1-4). This minimal delay referred to the time the animals spent on the platform in trial 1 of the test. In the "delay condition" (days 5-8), the animals were sacrificed for the purpose of immunohistochemical evaluation of BrdU and doublecortin $(D C X)$ expression in the dentate gyrus. b Schematic coronal brain sections delineating the hippocampal areas investigated with reference to bregma (adapted from The mouse brain in stereotaxic coordinates by Franklin and Paxinos 2008). Counting of BrdU and DCX immunoreactivity cells was performed in the dentate gyrus $(D G)$ region of the complete (i.e., dorsal plus ventral) hippocampus. All immunohistochemical analyses were performed on sections ranging from bregma -1.3 to $-3.4 \mathrm{~mm}$ according to The mouse brain in stereotaxic coordinates (Franklin and Paxinos 2008). $C A 1$ field CA1 hippocampus, CA3 field CA3 hippocampus

presented with an additional intertrial delay of $60 \mathrm{~s}$, during which they were kept in a waiting box filled with sawdust.

Escape latency and distance swum were calculated for each trial. The critical measure of working memory in this task is the reduction in time spent and/or distance swum to find the submerged platform in trial 2, compared with trial 1 (when the platform location was essentially unknown to the subjects). Data collection was achieved using the Ethovision tracking system (Noldus Information Technology, Wageningen, The Netherlands).

Immunohistochemistry

Two days after completion of the working memory test, all animals were sacrificed to perform the immunohistochemical investigations. The animals were anesthetized with an overdose of Nembutal ${ }^{\mathrm{TM}}$ (Abbott Laboratories, North Chicago, IL, USA) and perfused transcardially with $0.9 \%$ $\mathrm{NaCl}$, followed by $4 \%$ phosphate-buffered paraformaldehyde solution containing $15 \%$ picric acid. The dissected 
brains were postfixed in the same fixative for $6 \mathrm{~h}$ and processed for antigen retrieval involving overnight incubation in citric acid buffer $(\mathrm{pH}=4.5)$ followed by a $90-\mathrm{s}$ microwave treatment at $480 \mathrm{~W}$ (Fritschy et al. 1998). The brains were then cryoprotected using 30\% sucrose in phosphate buffered saline (PBS) and frozen with powdered dry ice.

Coronal sections ( $40 \mu \mathrm{m}$ thick) were cut from frozen blocks on a sliding microtome, and eight randomly sampled series of sections from Bregma -0.8 to $-3.6 \mathrm{~mm}$ were collected, rinsed in PBS, and stored at $-20^{\circ} \mathrm{C}$ in antifreeze solution until further processing. For immunostaining, the slices were first rinsed three times for $10 \mathrm{~min}$ in PBS. Blocking was done in PBS, $0.3 \%$ Triton $\mathrm{X}-100$, and $5 \%$ normal serum for $1 \mathrm{~h}$ at room temperature. The following primary antibodies were used: rat anti-BrdU (Oxford Biotechnology Ltd., Kidlington, UK; diluted 1:3,000) and horse anti-DCX (Santa Cruz Biotechnology, Santa Cruz, CA, USA; diluted 1:1,000). The primary antibodies were diluted in PBS containing 2\% normal goat serum and $0.3 \%$ Triton X-100 and incubated overnight at room temperature. After three washes with PBS, the sections were incubated with the biotinylated secondary goat antibody (Jackson ImmunoResearch Laboratories Inc., West Grove, PA, USA), diluted 1:500 in antibody buffer for $1 \mathrm{~h}$ at room temperature. The sections were washed again three times for $10 \mathrm{~min}$ in PBS and incubated with Vectastain Kit (Vector Laboratories; Burlingame, CA, USA) diluted in PBS for $1 \mathrm{~h}$. After three rinses in Tris- $\mathrm{HCl} 0.1 \mathrm{M}(\mathrm{pH}=$ 7.4 ), the sections were stained with $1.25 \%$ 3,3-diaminobenzidine and $0.08 \% \mathrm{H}_{2} \mathrm{O}_{2}$ for $10 \mathrm{~min}$, rinsed four times in PBS, dehydrated, and cover-slipped with Eukid ${ }^{\circledR}$ (Kindler GmbH, Freiburg, Germany).

\section{Counting of BrdU- and DCX-positive cells}

The total numbers of BrdU- and DCX-positive cells were estimated in the right dentate gyrus (DG), thereby including both dorsal and ventral parts. The DG was delineated according to The mouse brain in stereotaxic coordinates by Franklin and Paxinos (2008). Counting of BrdU- and DCXpositive cells was conducted on brain sections ranging from Bregma -1.3 to $-3.4 \mathrm{~mm}$ according to The mouse brain in stereotaxic coordinates (Franklin and Paxinos 2008). Schematic coronal brain sections of the hippocampal area of interest with reference to Bregma are provided in Fig. $1 b$.

For each animal, every section out of a one-in-eight series was measured, resulting in an average of six sections per animal. The majority of newborn (immature) neurons in the DG resides in a narrow band of tissue, namely the subgranular zone (SGZ). Since the SGZ is only about two to three nuclei wide (Kralic et al. 2005; Rahimi and
Claiborne 2007; see also Figs. 3 and 4), BrdU- and DCXpositive cells were counted exhaustively using live microscopy (Zeiss Axioplan microscope, Zeiss, Jena, Germany) with a $40 \times$ objective (numerical aperture, 0.45). Every labeled cell within the outlined DG of the right brain hemisphere was counted using a marker tool in Stereo Investigator (version 6.50.1, Microbrightfield, Colchester, VT, USA). The total number of BrdU- and DCX-positive cells in the DG was estimated according to the formula: Cells $_{\mathrm{Tot}}=\sum$ Cells $\times 1 /$ ssf, where $\mathrm{ssf}=$ section sampling fraction (1/8). For the analysis, total estimated BrdU- and DCX-positive cells per unit volume (cells per cubic millimeter) in the SGZ and the granule cell layer (GCL) of the DG were taken into account for each animal. Counting of cells was performed blind to the animals' prenatal treatment and adult drug conditions.

\section{Statistical analysis}

All data were analyzed using parametric ANOVA. In the working memory test, the latency and distance swum to find the submerged platform were analyzed with the between-subjects factors of prenatal treatment and adult drug treatment and with the within-subjects factors of trials and days. The data collected on the two phases of the working memory test (i.e., in the "minimal delay condition" and " 60 -s delay condition") were analyzed separately. Hence, a $2 \times 2 \times 2 \times 4$ (prenatal treatment $\times$ antipsychotic drug treatment $\times$ trials $\times$ days) ANOVA of repeated measures was used for both delay conditions. Whenever appropriate, additional $2 \times 4$ (trials $\times$ days) ANOVAs restricted to each of the four experimental groups were used to verify the presence of a significant trial effect in each group. Estimates of BrdU- and DCX-positive cells in the DG were analyzed using a $2 \times 2$ (prenatal treatment $\times$ adult drug treatment) ANOVA. Statistical significance was set at $p<$ 0.05 . All statistical analyses were conducted using the statistical software StatView (version 5.0) implemented on a personal computer running the Windows XP operating system.

\section{Results}

Effects of prenatal immune challenge and chronic clozapine treatment on spatial working memory

Pretraining Animals from all experimental groups were able to locate the visible platform positioned in the center of the maze during the 1-day pretraining session. However, one animal from the VEH-treated PolyI:C group and one animal from the CLZ-treated PolyI:C group displayed excessive floating behavior. These two animals were 
therefore excluded from the subsequent working memory task and immunohistochemical investigations.

Minimal delay condition No group differences were detected in the latency and distance swum to find the submerged platform from trial 1 (when the location of the platform was essentially unknown to the animals) to trial 2 when the animals were tested using a minimal (10-15 s) intertrial delay (Fig. 2a). For all groups, the latency and distance swum to find the platform was reduced in trial 2 relative to trial 1 (Fig. 2a), indicating intact spatial working memory when tested under the minimal delay condition. ANOVA of escape latency and distance swum only revealed a highly significant main effect of trials (latency: $F(1,26)=28.9, p<0.001$; distance: $F(1,26)=37.8$, $p<0.001)$. No interactions involving the between-subjects factors of prenatal treatment and/or adult drug treatment attained statistical significance.

Sixty-second delay condition Prenatal PolyI:C-induced immune challenge led to impaired working memory performance when the interval between trials 1 and 2 on each of the subsequent four test days was increased to $60 \mathrm{~s}$. Importantly, the prenatal PolyI:C-induced working memory deficit was normalized by chronic CLZ treatment. Hence, while CLZ-treated PolyI:C offspring displayed a clear reduction in the latency and distance swum from trials 1 to 2 under the 60-s delay condition, VEH-treated PolyI:C offspring did not (Fig. 2b). Interestingly, chronic CLZ treatment exerted opposite effects on working memory performance in offspring born to control mothers: While VEH-exposed control offspring also showed a significant reduction in the latency and distance swum from trials 1 to 2, CLZ-treated control offspring were similar to VEHtreated PolyI:C offspring in failing to show a comparable improvement (Fig. 2b).

Statistical support for these impressions was yielded by the significant three-way interaction between prenatal treatment, adult drug treatment, and trials in the ANOVA of escape latency $(F(1,26)=4.2, p<0.05)$ and distance swum $(F(1,26)=4.4, p<0.05)$. Subsequent analyses restricted to each experimental group were then conducted in order to verify the presence of a significant trial effect during the 4 days of testing under the 60 -s delay condition. These analyses confirmed the significant reduction in time spent and distance swum to find the platform in trial 2 relative to trial 1 in both VEH-treated control offspring (main effect of trials for latency: $F(1,21)=8.1, p<0.05$; main effect of trials for distance: $F(1,21)=7.4, p<0.05)$ and CLZ-treated PolyI:C offspring (main effect of trials for latency: $F(1,18)=16.8, p<0.01$; main effect of trials for distance: $F(1,18)=15.1, p<0.01)$, but not in the other two groups $(F$ 's $<1$, n.s. $)$.
Effects of prenatal immune challenge and chronic CLZ treatment on BrdU-positive cells in the DG

BrdU-positive cells were clearly identifiable in both the dorsal and ventral hippocampus by the presence of darkly stained cell bodies (see Fig. 3a). Consistent with the neurogenic capacity of the DG in the adult HPC (Balu and Lucki 2009), BrdU-immunoreactive cells were primarily found in the DG region. Most importantly, adult offspring born to gestationally immune-challenged mothers showed a significant reduction in the number of BrdU-positive cells in the DG compared to adult control offspring (Fig. 3b). The prenatal infection-induced decrease in BrdU-immunoreactive cells was noticeable in both CLZ- and VEH-treated subjects, indicating that chronic CLZ administration did not exert any influence on the number of BrdU-positive cells in offspring born to immune-challenged mothers (Fig. 3b). Similarly, chronic CLZ treatment did also not significantly affect the number of BrdU-immunoreactive cells in adult offspring born to control mothers relative to VEH treatment in corresponding control offspring (Fig. 3b). Statistical support for these impression was obtained by the ANOVA of BrdU-positive cells in the DG, which revealed only a significant main effect of prenatal treatment $(F(1,26)=8.93, p<0.01)$.

Effects of prenatal immune challenge and chronic CLZ treatment on DCX-positive cells in the DG

DCX-positive cells were also clearly identifiable in the DG region of the dorsal and ventral hippocampal formation. Darkly stained DCX-positive cell bodies were found primarily in the SGZ of the DG and, to a lesser extent, in the outer zones of the GCL (see Fig. 4a). The DCX-positive cells in the SGZ and GCL also displayed the characteristic dendritic trees extending into the inner molecular layer (see Fig. 4a).

Consistent with the results obtained in the analysis of BrdU-positive cells (see Fig. 3), adult offspring born to gestationally immune-challenged mothers showed a marked decrease in the number of DCX-positive cells in the DG compared to adult control offspring (Fig. 4b). Again, chronic CLZ treatment did not exert any significant influence on the number of DCX-positive cells regardless of the prenatal immunological treatment (Fig. 4b). The numbers of DCX-positive cells were thus highly comparable between VEH- and CLZ-treated control offspring and between VEH- and CLZ-treated PolyI:C offspring (Fig. 4). Statistical support for this impressions was yielded by the ANOVA of DCX-positive cells in the DG, which revealed only a significant main effect of prenatal treatment $(F(1$, 26) $=4.79, p<0.05)$. 
a
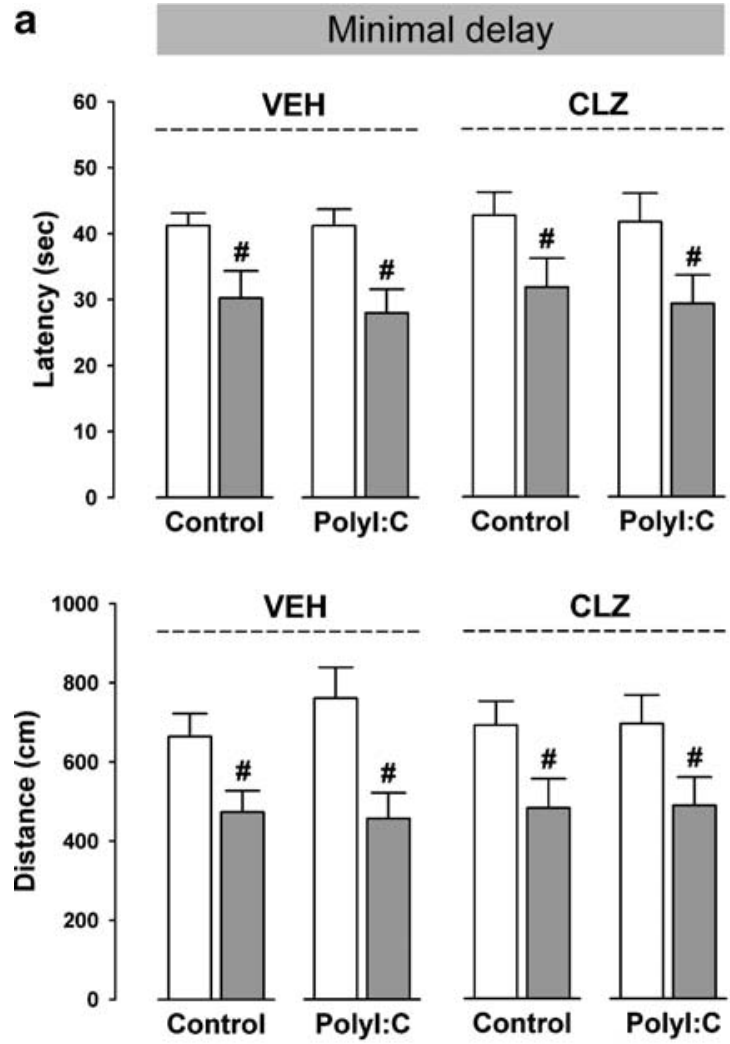

Trial 1

Trial 2
Fig. 2 Effects of prenatal immune activation and chronic clozapine $(C L Z)$ treatment on spatial working memory in a matching-to-position water maze task. The graph depicts the latency and distance swum to find the submerged platform in trial 1 (when the location of the platform was unknown to the animals) and in trial 2. Working memory was indexed by the reduction in the latency and distance swum from trial 1 to trial 2. a Neither prenatal immune challenge by the viral mimic PolyI:C nor chronic treatment with the atypical antipsychotic drug CLZ significantly affected working memory performance when the animals were tested in trials presented with a minimal (10-15 s) interval between trials 1 and 2. Hence, a clear reduction in the latency and distance swum to find the platform in trial 2 relative to trial 1 was noticeable in both CLZ- or vehicle $(V E H)$-treated PolyI:C and control

\section{Discussion}

The present study sought evidence for the hypothesis that chronic treatment with the atypical antipsychotic drug CLZ may improve prenatal infection-induced cognitive dysfunctions by stimulating adult hippocampal neurogenesis. Using a well-established mouse model of prenatal immune challenge by the viral mimic PolyI:C (Meyer et al. 2009a, b), our results do not support this hypothesis. Consistent with the earlier reports of beneficial effects of CLZ on other prenatal infection-induced behavioral and cognitive abnormalities (Shi et al. 2003; Zuckerman et al. 2003; Zuckerman and Weiner 2005; Ozawa et al. 2006), we found that chronic CLZ treatment significantly improved spatial working memory deficits in adult offspring born to b

60-sec delay
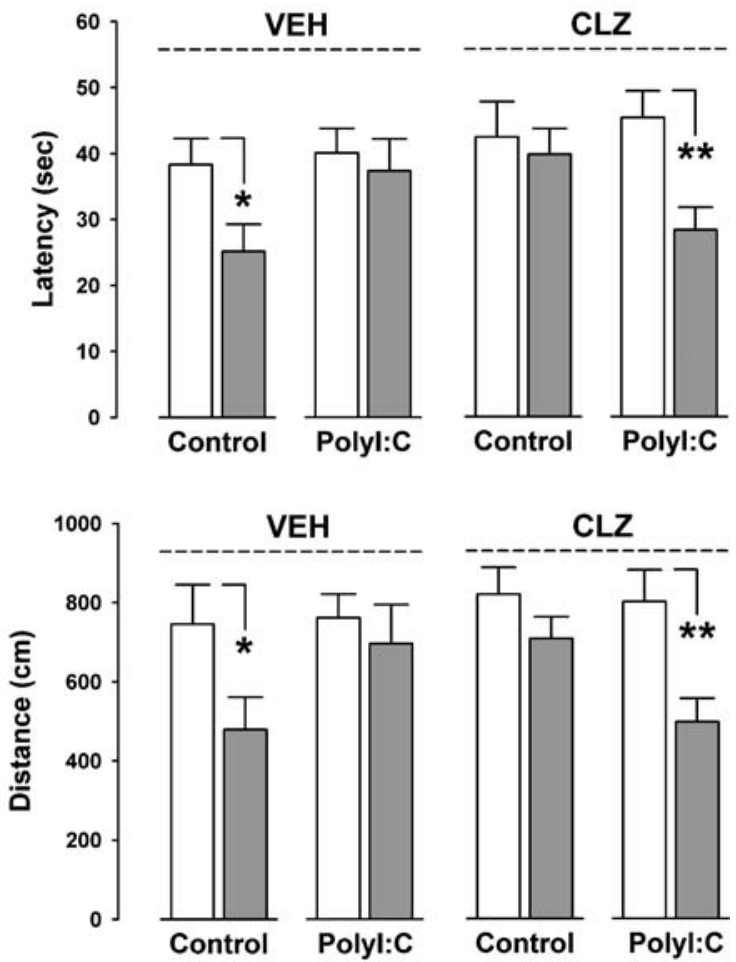

Trial 1

offspring. $\# p<0.001$ refers to a statistical significance associated with the main effect of trials taken from the corresponding ANOVA. b When the interval between trials 1 and 2 was extended to $60 \mathrm{~s}$, working memory was markedly impaired in VEH-treated PolyI:C offspring and in CLZ-treated control offspring. Only VEH-treated control offspring and CLZ-treated PolyI:C offspring displayed a significant reduction in the latency and distance swum in trial 2 relative to trial 1. VEH-treated PolyI:C offspring and CLZ-treated control offspring did not show a similar improvement. ${ }^{*} p<0.05$ and $*^{*} p<0.01$ refer to a statistical significance associated with the significant main effect of trials in the ANOVA restricted to each experimental group. All values are means \pm SEM

immune-challenged mothers. However, these procognitive effects of the drug treatment were not related to concomitant effects on adult hippocampal neurogenesis. Hence, adult offspring born to PolyI:C-exposed mothers displayed a significant reduction in BrdU- and DCX-immunoreactive cells regardless of whether they were subjected to chronic CLZ treatment or not. In accordance with the drug's null effect on adult hippocampal neurogenesis in prenatally immune challenged animals, chronic CLZ treatment did also not significantly affect the expression of cellular markers of adult neurogenesis in adult control offspring (Figs. 3 and 4). However, the chronic drug regime negatively affected working memory performance in adult offspring born to control mothers. Taken together, our findings thus suggest that chronic CLZ treatment can have 
a

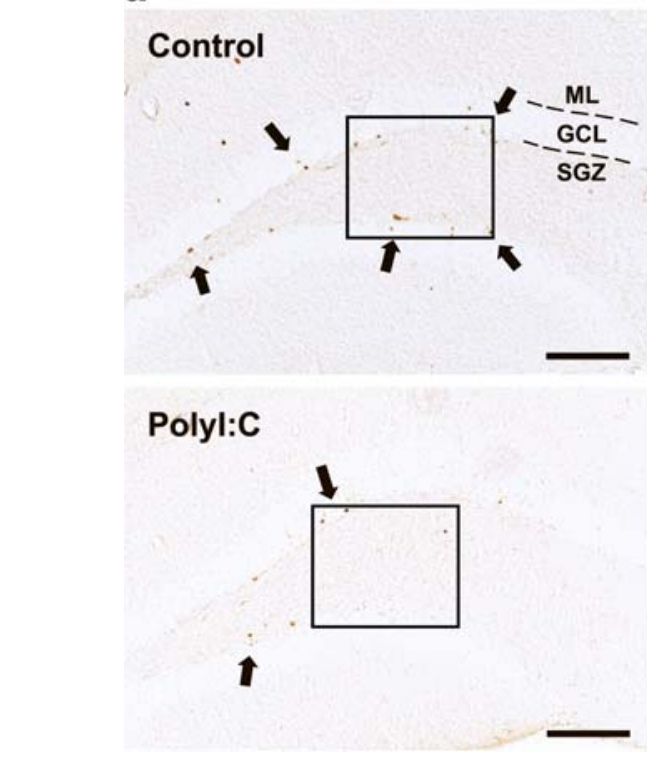

Fig. 3 Bromodeoxyuridine $(B r d U)$ immunoreactivity in the dentate gyrus of adult offspring born to PolyI:C-exposed or control mothers. a The photomicrographs show coronal brain sections of representative adult control and PolyI:C offspring displaying high and low numbers of BrdU-positive cells (indicated by the arrows), respectively, in the dentate gyrus at the level of the dorsal hippocampal formation. As evident in the images at a higher magnification (indicated by the squares), darkly stained BrdU-positive cell bodies were found primarily in the subgranular zone $(S G Z)$ and, to a notably lesser extent, in the granular cell layer $(G C L)$ and molecular layer $(M L)$ of

bidirectional effects on working memory performance depending on whether the treated subjects were predisposed to adult neuropathology by prenatal immune challenge or not and that this causal relationship is independent of adult hippocampal neurogenesis.

In full agreement with our previous findings (Meyer et al. 2005, 2008b), the prenatal infection-induced working memory impairment was only apparent in conditions, in which the demand in temporal retention was accentuated by increasing the delay between the two relevant test trials: All animals showed normal working memory performance when tested under the minimal delay condition (10-15 s); however, a significant impairment was evident in prenatally PolyI:C-exposed offspring when the delay between trials 1 and 2 of the working memory test was extended to $60 \mathrm{~s}$ (Fig. 2). This indicates that the working memory impairment in prenatally immune-challenged subjects may be accounted for by deficits in mnemonic processing (Olton et al. 1986; Delatour and Gisquet-Verrier 1999; Broersen 2000). According to this interpretation, working memory is normal under low working memory demand, but significant impairment emerges when the mnemonic flexibility required by the working memory task is accentuated by additional storage load in the temporal domain. Interestingly, the CLZ-induced disruption of working memory in b

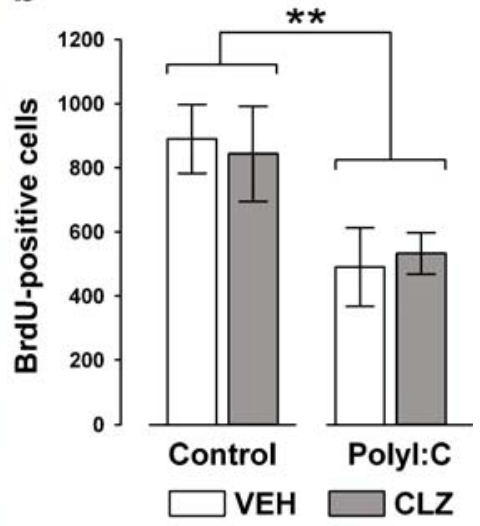

the dentate gyrus. Scale bars $=250 \mu \mathrm{m}$. b Adult offspring born to PolyI:C-treated mothers showed a significant reduction in the number of BrdU-positive cells in the dentate gyrus compared to adult offspring born to control mothers. Chronic clozapine (CLZ) treatment did not significantly influence the number of BrdU-positive cells compared with corresponding vehicle $(\mathrm{VEH})$ treatment in adult control or PolyI:C offspring. $* p<0.05$ refers to a statistical significance associated with the main effect of prenatal treatment in the corresponding ANOVA. All values are means $\pm \mathrm{SEM}$

offspring born to control mothers was also only evident in conditions, in which the demand in temporal retention was accentuated by increasing the delay between the two relevant test trials (Fig. 2). One possible explanation would therefore be that the CLZ-induced disruption of working memory in offspring born to control mothers could be related to drug-induced changes in mnemonic functions.

The present findings of bidirectional cognitive effects of chronic CLZ treatment are striking but not unprecedented. Indeed, previous experimental research in rats has already provided direct evidence that the outcomes of therapeutic CLZ treatment on working memory performance can critically differ between intact control subjects and subjects with preexisting brain lesions, especially within the hippocampal formation. For example, CLZ treatment is efficient in improving working memory deficits in rats with fimbria-fornix lesions, while at the same time it impairs working memory in sham-operated control rats (Addy et al. 2005). Likewise, CLZ treatment at doses that lead to significant working memory impairment in intact animals significantly improves working memory deficits induced by chronic blockade of hippocampal $\alpha 4 \beta 2$ nicotinic receptors (Pocivavsek et al. 2006). In addition to the deficits in adult hippocampal neurogenesis reported here, prenatal immune activation in rodents is known to result in a wide spectrum 
a

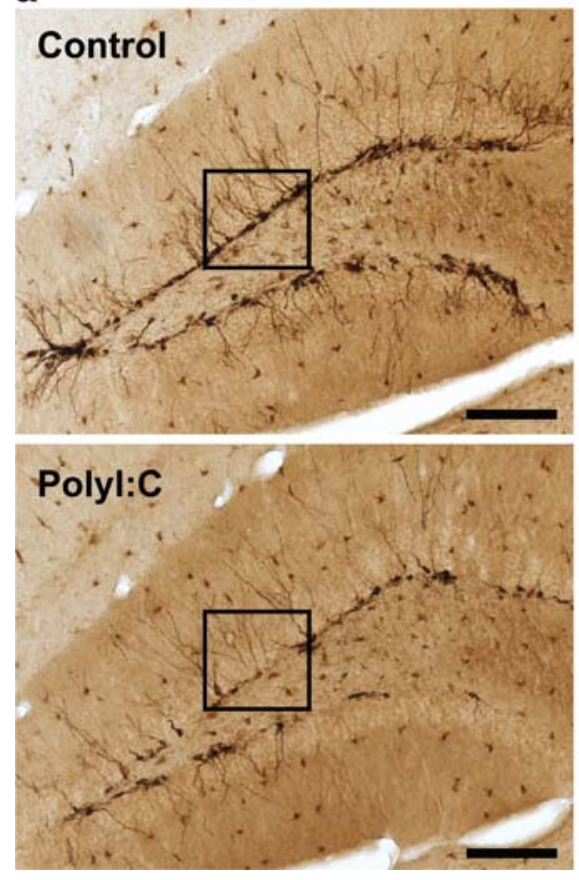

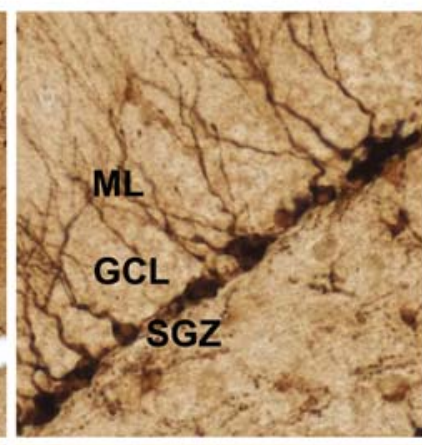

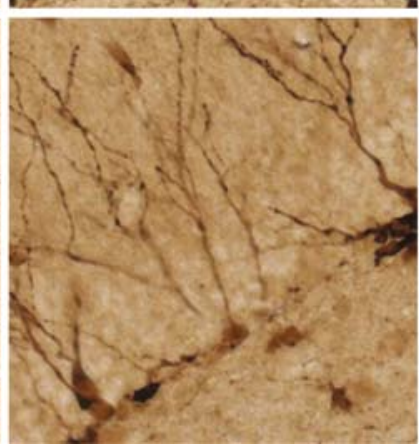

b

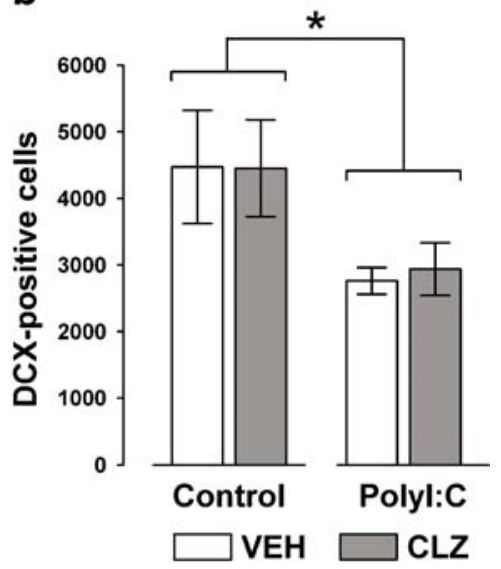

Fig. 4 Doublecortin $(D C X)$ immunoreactivity in the dentate gyrus of adult offspring born to PolyI:C-exposed or control mothers. a The photomicrographs show coronal brain sections of representative adult control and PolyI:C offspring displaying high and low numbers of DCX-positive cells, respectively, in the dentate gyrus at the level of the dorsal hippocampal formation. As evident in the images at a higher magnification (indicated by the squares), darkly stained DCX-positive cell bodies were found primarily in the subgranular zone (SGZ). These DCX-positive cells extend characteristic dendritic trees through the granular cell layer $(G C L)$ into the inner molecular layer $(M L)$ of the dentate gyrus. Scale bars $=250 \mu \mathrm{m}$. b Adult offspring born to PolyI:Ctreated mothers showed a significant reduction in the number of DCXpositive cells in the dentate gyrus compared to adult offspring born to control mothers. Chronic clozapine $(C L Z)$ treatment did not significantly influence the number of DCX-positive cells compared with corresponding vehicle $(V E H)$ treatment in adult control or PolyI:C offspring. ${ }^{*} p<0.05$ refers to a statistical significance associated with the main effect of prenatal treatment in the corresponding ANOVA. All values are means \pm SEM

Weinberger 1999; Antonova et al. 2004; Tamminga 2006), the nature and/or severity of hippocampal abnormalities are likely to differ considerably between individual subjects suffering from this disorder. Varying degrees of hippocampal dysfunction may thus not only determine the specificity of symptom clusters in schizophrenia (O'Donnell and Grace 1998) but also critically influence the effects of atypical antipsychotic drug treatment on behavioral and cognitive symptoms, including working memory.

One of the main findings of the present study is that prenatal exposure to a viral-like acute phase response leads to long-term deficits in adult hippocampal neurogenesis. This extends our previous report of deficient hippocampal neurogenesis in juvenile offspring born to gestationally PolyI:C-exposed mice (Meyer et al. 2006). Furthermore, the neurogenesis-disrupting effects of prenatal viral-like immune activation reported here are in agreement with the recent experimental data obtained in a rat model of prenatal immune challenge induced by the bacterial endotoxin LPS (Cui et al. 2009). Hence, various types of immuneassociated prenatal insults can induce robust deficits in pathophysiology of schizophrenia (Harrison 1999, 2004; 
postnatal hippocampal neurogenesis across juvenile to adult stages of development.

In the present study, adult hippocampal neurogenesis was studied by postmortem investigations of BrdU and DCX immunoreactivity in the DG. Antibodies directed against the microtubule-associated protein DCX have been consistently shown to label newly generated postmitotic neurons before they reach full maturation (Francis et al. 1999; Brown et al. 2003; Couillard-Despres et al. 2005). DCX is thus a valuable marker to investigate aspects of neurogenesis which are associated primarily with the developmental processes occurring between neuronal differentiation and maturation. The prenatal PolyI:C-induced deficit in hippocampal DCX immunoreactivity (Fig. 4) can thus be interpreted as a form of reduced neuronal differentiation and/or maturation. In addition, we showed reduced cell labeling of the mitosis marker BrdU in the DG of prenatally immune-challenged offspring relative to control offspring (Fig. 3). Our experimental design required that the animals were sacrificed 10 days following the last BrdU injection (see Fig. 1). According to current consensus (Kempermann et al. 2004; Balu and Lucki 2009), evaluation of BrdU-positive cells following such an incubation interval would provide information about the integration of newly born cells into the hippocampal formation. Hence, our analysis of BrdU-positive cells suggests that the prenatal infection-induced deficit in neuronal differentiation and/or maturation (as assessed by DCX immunostaining) may stem from diminished neuronal cell integration in the adult DG (Kempermann et al. 2004; Balu and Lucki 2009). We cannot further conclude from the present results as to whether these deficits may additionally be related to reduced cell proliferation in the adult DG of prenatally immune-challenged animals. This is because we did not assess BrdU-positive cells using a short-term (2-24 h) interval between BrdU administration and sacrifice of the animals. However, recent experimental evidence suggests that prenatal immune activation is indeed effective in reducing the capacity of postnatal hippocampal cell proliferation as assessed by quantification of BrdU-positive cells in the DG shortly ( $2 \mathrm{~h}$ ) after BrdU administration (Cui et al. 2009).

The lack of a significant effect of chronic CLZ treatment on hippocampal neurogenesis reported here is in agreement with previous experimental studies showing no significant effects of acute or chronic treatment with low or high doses of CLZ on adult hippocampal neurogenesis in rats (Halim et al. 2004; Schmitt et al. 2004). Since the chosen dose of CLZ $(5 \mathrm{mg} / \mathrm{kg} /$ day) proved to be functionally effective in the assessment of cognitive functions (Fig. 2), it is unlikely that the null effect of chronic CLZ treatment on adult hippocampal neurogenesis may be explained by a general lack of drug effect. Rather, our findings are indicative of a critical segregation between the effects of chronic CLZ on cognitive functions and adult hippocampal neurogenesis. This segregation is strikingly similar to recent experimental findings in rats suggesting that the therapeutic efficacy of antidepressant drugs to ameliorate depression-like behavioral abnormalities can be segregated from the drugs' effects on adult hippocampal neurogenesis (Bessa et al. 2009a, b). However, in contrast to our findings here and the earlier reports in rats (Halim et al. 2004; Schmitt et al. 2004), a recent study in mice has shown that chronic CLZ treatment is able to prevent a decrease in adult hippocampal neurogenesis (as assessed by BrdU labeling in the DG) induced by repeated administration of the noncompetitive NMDA-receptor antagonist phencyclidine (PCP; Maeda et al. 2007). This suggests that even though chronic CLZ administration may not stimulate adult hippocampal neurogenesis per se, it may prevent neurogenesis-disrupting effects induced by specific (experimental) manipulations. It is important to emphasize that the PCP-induced impairment in adult hippocampal neurogenesis reported by Maeda et al. (2007) critically differs from that induced by prenatal immune challenge: The former is acquired during adult life, while the latter is present already at juvenile stages of life (Meyer et al. 2006; Cui et al. 2009). Hence, the efficacy of CLZ to prevent (experimentally induced) impairments in adult hippocampal neurogenesis may crucially depend on the precise developmental window at which the deficit is induced.

Notably, the significant effects of the prenatal immunological and adult pharmacological manipulations on spatial working memory in the water maze clearly dissociated from their effects on adult hippocampal neurogenesis. Indeed, while both manipulations exerted a significant (bidirectional) influence on working memory (see Fig. 2), only prenatal PolyI:C-induced immune activation but not chronic CLZ treatment significantly affected the numbers of BrdU- and DCX-positive cells in the adult DG (Figs. 3 and 4). This dissociation may indicate that adult neurogenesis is not essentially involved in spatial working memory, at least when assessed in the Morris water maze. This suggestion is consistent with the findings from numerous attempts to explore the functional role of the adult DG in general (Shapiro and Olton 1994; Gilbert et al. 1998, 2001; Kesner 2007; Goodrich-Hunsaker et al. 2008) and of hippocampal neurogenesis in particular (Clelland et al. 2009). More specifically, there is experimental evidence to assume that the contribution of the DG to cognitive functions may be more directly related to spatial (and temporal) separation of events associated with transient memory representations of new spatial information, rather than to (spatial) working memory representations of new information (Gilbert et al. 1998, 2001; Leutgeb et al. 2007). Of special interest in the present context are the recent 
findings by Clelland et al. (2009) showing that ablation of hippocampal neurogenesis in adult mice leads to impaired spatial memory for similar, but not distinct, spatial locations, emphasizing a crucial role of adult hippocampal neurogenesis to spatial pattern separation. Given these novel findings, it would be highly interesting to further explore the functional role of prenatal infection-induced impairments in hippocampal neurogenesis in spatial pattern separation.

In conclusion, the present study demonstrates that chronic treatment with the reference atypical antipsychotic drug CLZ significantly influences working memory performance without affecting hippocampal neurogenesis in adulthood. Hence, the beneficial effects of chronic CLZ on working memory performance in neurodevelopmentally comprised subjects do not seem to be casually linked to concomitant effects on adult hippocampal neurogenesis. With respect to the clinical situation in humans, our experimental findings thus provide no support for the recent hypothesis that the beneficial effects of atypical antipsychotic drugs on working memory dysfunctions in patients with schizophrenia may be mediated by a reinstatement of deficient hippocampal neurogenesis (Reif et al. 2006, 2007; Toro and Deakin 2007). Additional investigations are thus clearly warranted in order to identify the critical neuropharmacological and cellular mechanisms underlying the procognitive effects of CLZ treatment, in general, and in subjects predisposed to adult neuropathology by prenatal immune challenge, in particular.

\begin{abstract}
Acknowledgments The research presented in this study was partially supported by The Swiss National Science Foundation (SNSF) grant 310000-118284/1, by The Swiss Federal Institute of Technology (ETH Zurich) grant-11 07/03, and by a 2009 NARSAD Distinguished Investigator Award to Joram Feldon. We are extremely grateful to the Animal Services Department Schwerzenbach, Swiss Federal Institute of Technology, for their excellent animal husbandry and care.
\end{abstract}

Disclosure All authors declare they have no conflicts of interest to disclose.

\section{References}

Addy NA, Pocivavsek A, Levin ED (2005) Reversal of clozapine effects on working memory in rats with fimbria-fornix lesions. Neuropsychopharmacology 30:1121-1127

Adler G, Grieshaber S, Faude V, Thebaldi B, Dressing H (2002) Clozapine in patients with chronic schizophrenia: serum level, EEG and memory performance. Pharmacopsychiatry 35:190-194

Altman J, Das GD (1965) Post-natal origin of microneurones in the rat brain. Nature 207:953-956

Antonova E, Sharma T, Morris R, Kumari V (2004) The relationship between brain structure and neurocognition in schizophrenia: a selective review. Schizophr Res 70:117-145

Baddeley A (2003) Working memory: looking back and looking forward. Nat Rev Neurosci 4:829-839
Balu DT, Lucki I (2009) Adult hippocampal neurogenesis: regulation, functional implications, and contribution to disease pathology. Neurosci Biobehav Rev 33:232-252

Bannerman DM, Rawlins JN, McHugh SB, Deacon RM, Yee BK, Bast T, Zhang WN, Pothuizen HH, Feldon J (2004) Regional dissociations within the hippocampus-memory and anxiety. Neurosci Biobehav Rev 28:273-283

Bessa JM, Ferreira D, Melo I, Marques F, Cerqueira JJ, Palha JA, Almeida OF, Sousa N (2009a) The mood-improving actions of antidepressants do not depend on neurogenesis but are associated with neuronal remodeling. Mol Psychiatry 14:764-773

Bessa JM, Ferreira D, Melo I, Marques F, Cerqueira JJ, Palha JA, Almeida OF, Sousa N (2009b) Hippocampal neurogenesis induced by antidepressant drugs: an epiphenomenon in their mood-improving actions. Mol Psychiatry 14:739

Broersen LM (2000) Attentional processes and learning and memory in rats: the prefrontal cortex and hippocampus compared. Prog Brain Res 126:79-94

Brown AS (2006) Prenatal infection as a risk factor for schizophrenia. Schizophr Bull 32:200-202

Brown AS, Susser ES (2002) In utero infection and adult schizophrenia. Ment Retard Dev Disabil Res Rev 8:51-57

Brown JP, Couillard-Després S, Cooper-Kuhn CM, Winkler J, Aigner L, Kuhn HG (2003) Transient expression of doublecortin during adult neurogenesis. J Comp Neurol 467:1-10

Brown AS, Vinogradov S, Kremen WS, Poole JH, Deicken RF, Penner JD, McKeague IW, Kochetkova A, Kern D, Schaefer CA (2009) Prenatal exposure to maternal infection and executive dysfunction in adult schizophrenia. Am J Psychiatry 166:683690

Cardon M, Ron-Harel N, Cohen H, Lewitus GM, Schwartz M (2009) Dysregulation of kisspeptin and neurogenesis at adolescence link inborn immune deficits to the late onset of abnormal sensorimotor gating in congenital psychological disorders. Mol Psychiatry (in press)

Clancy B, Darlington RB, Finlay BL (2001) Translating developmental time across mammalian species. Neuroscience 105:7-17

Clancy B, Kersh B, Hyde J, Darlington RB, Anand KJ, Finlay BL (2007) Web-based method for translating neurodevelopment from laboratory species to humans. Neuroinformatics 5:79-94

Clelland CD, Choi M, Romberg C, Clemenson GD Jr, Fragniere A, Tyers P, Jessberger S, Saksida LM, Barker RA, Gage FH, Bussey TJ (2009) A functional role for adult hippocampal neurogenesis in spatial pattern separation. Science 325:210-213

Couillard-Despres S, Winner B, Schaubeck S, Aigner R, Vroemen M, Weidner N, Bogdahn U, Winkler J, Kuhn HG, Aigner L (2005) Doublecortin expression levels in adult brain reflect neurogenesis. Eur J Neurosci 21:1-14

Coyle P, Tran N, Fung JN, Summers BL, Rofe AM (2009) Maternal dietary zinc supplementation prevents aberrant behaviour in an object recognition task in mice offspring exposed to LPS in early pregnancy. Behav Brain Res 197:210-218

Cui K, Ashdown H, Luheshi GN, Boksa P (2009) Effects of prenatal immune activation on hippocampal neurogenesis in the rat. Schizophr Res 113:288-297

Delatour B, Gisquet-Verrier P (1999) Lesions of the prelimbicinfralimbic cortices in rats do not disrupt response selection processes but induce delay-dependent deficits: evidence for a role in working memory? Behav Neurosci 113:941-955

Dolbeare F (1995) Bromodeoxyuridine: a diagnostic tool in biology and medicine, part I: historical perspectives, histochemical methods and cell kinetics. Histochem J 27:339-369

Fatemi SH (2005) Neuropsychiatric disorders and infection. Taylor \& Francis, London

Francis F, Koulakoff A, Boucher D, Chafey P, Schaar B, Vinet MC, Friocourt G, McDonnell N, Reiner O, Kahn A, McConnell SK, 
Berwald-Netter Y, Denoulet P, Chelly J (1999) Doublecortin is a developmentally regulated, microtubule-associated protein expressed in migrating and differentiating neurons. Neuron 23:247-256

Franklin KBJ, Paxinos G (2008) The mouse brain in stereotaxic coordinates. Elsevier, Amsterdam

Fritschy JM, Weinmann O, Wenzel A, Benke D (1998) Synapsespecific localization of NMDA and GABA(A) receptor subunits revealed by antigen-retrieval immunohistochemistry. J Comp Neurol 390:194-210

Galletly CA, Clark CR, McFarlane AC, Weber DL (1997) Relationships between changes in symptoms ratings, neuropsychological test performance, and quality of life in schizophrenic patients with clozapine. Psychiatry Res 72:161-166

Galletly CA, Clark CR, McFarlane AC (2005) Clozapine improves working memory updating in schizophrenia. Eur Neuropsychopharmacol 15:601-608

Gilbert PE, Kesner RP, DeCoteau WE (1998) Memory for spatial location: role of the hippocampus in mediating spatial pattern separation. J Neurosci 18:804-810

Gilbert PE, Kesner RP, Lee I (2001) Dissociating hippocampal subregions: double dissociation between dentate gyrus and CA1. Hippocampus 11:626-636

Gilmore JH, Jarskog LF (1997) Exposure to infection and brain development: cytokines in the pathogenesis of schizophrenia. Schizophr Res 24:365-367

Goodrich-Hunsaker NJ, Hunsaker MR, Kesner RP (2008) The interactions and dissociations of the dorsal hippocampus subregions: how the dentate gyrus, $\mathrm{CA} 3$, and CA1 process spatial information. Behav Neurosci 122:16-26

Grace J, Bellus SB, Raulin ML, Herz MI, Priest BL, Brenner V, Donnelly K, Smith P, Gunn S (1996) Long-term impact of clozapine and psychosocial treatment on psychiatric symptoms and cognitive functioning. Psychiatr Serv 47:41-45

Hagger C, Buckley P, Kenny JT, Friedman L, Ubogy D, Meltzer HY (1993) Improvement in cognitive functions and psychiatric symptoms in treatment-refractory schizophrenic patients receiving clozapine. Biol Psychiatry 34:702-712

Halim ND, Weickert CS, McClintock BW, Weinberger DR, Lipska BK (2004) Effects of chronic haloperidol and clozapine treatment on neurogenesis in the adult rat hippocampus. Neuropsychopharmacology 29:1063-1069

Harrison PJ (1999) The neuropathology of schizophrenia. A critical review of the data and their interpretation. Brain 122:593-624

Harrison PJ (2004) The hippocampus in schizophrenia: a review of the neuropathological evidence and its pathophysiological implications. Psychopharmacology 174:151-162

Kempermann G, Jessberger S, Steiner B, Kronenberg G (2004) Milestones of neuronal development in the adult hippocampus. Trends Neurosci 27:447-452

Kempermann G, Krebs J, Fabel K (2008) The contribution of failing adult hippocampal neurogenesis to psychiatric disorders. Curr Opin Psychiatry 21:290-295

Kesner RP (2007) A behavioral analysis of dentate gyrus function. Prog Brain Res 163:567-576

Kralic JE, Ledergerber DA, Fritschy JM (2005) Disruption of the neurogenic potential of the dentate gyrus in a mouse model of temporal lobe epilepsy with focal seizures. Eur J Neurosci 22:1916-1927

Lee I, Kesner RP (2003) Time-dependent relationship between the dorsal hippocampus and the prefrontal cortex in spatial memory. J Neurosci 23:1517-1523

Lee MA, Jayathilake K, Meltzer HY (1999) A comparison of the effect of clozapine with typical neuroleptics on cognitive function in neuroleptic-responsive schizophrenia. Schizophr Res 37:1-11
Leutgeb JK, Leutgeb S, Moser MB, Moser EI (2007) Pattern separation in the dentate gyrus and CA3 of the hippocampus. Science 315:961-966

Maeda K, Sugino H, Hirose T, Kitagawa H, Nagai T, Mizoguchi H, Takuma K, Yamada K (2007) Clozapine prevents a decrease in neurogenesis in mice repeatedly treated with phencyclidine. $\mathrm{J}$ Pharmacol Sci 103:299-308

McGurk SR, Carter C, Goldman R, Green MF, Marder SR, Xie H, Schooler NR, Kane JM (2005) The effects of clozapine and risperidone on spatial working memory in schizophrenia. Am J Psychiatry 162:1013-1036

Meyer U, Feldon J (2009) Neural basis of psychosis-related behaviour in the infection model of schizophrenia. Behav Brain Res 204:322-334

Meyer U, Feldon J, Schedlowski M, Yee BK (2005) Towards an immuno-precipitated neurodevelopmental animal model of schizophrenia. Neurosci Biobehav Rev 29:913-947

Meyer U, Nyffeler M, Engler A, Urwyler A, Schedlowski M, Knuesel I, Yee BK, Feldon J (2006) The time of prenatal immune challenge determines the specificity of inflammationmediated brain and behavioral pathology. J Neurosci 26:47524762

Meyer U, Yee BK, Feldon J (2007) The neurodevelopmental impact of prenatal infections at different times of pregnancy: the earlier the worse? Neuroscientist 13:241-256

Meyer U, Engler A, Weber L, Schedlowski M, Feldon J (2008a) Preliminary evidence for a modulation of fetal dopaminergic development by maternal immune activation during pregnancy. Neuroscience 154:701-709

Meyer U, Nyffeler M, Yee BK, Knuesel I, Feldon J (2008b) Adult brain and behavioral pathological markers of prenatal immune challenge during early/middle and late fetal development in mice. Brain Behav Immun 22:469-486

Meyer U, Feldon J, Fatemi SH (2009a) In-vivo rodent models for the experimental investigation of prenatal immune activation effects in neurodevelopmental brain disorders. Neurosci Biobehav Rev 33:1061-1079

Meyer U, Feldon J, Yee BK (2009b) A review of the fetal brain cytokine imbalance hypothesis of schizophrenia. Schizophr Bull 35:959-972

Moser E, Moser MB, Andersen P (1993) Spatial learning impairment parallels the magnitude of dorsal hippocampal lesions, but is hardly present following ventral lesions. J Neurosci 13:39163925

O'Donnell P, Grace AA (1998) Dysfunctions in multiple interrelated systems as the neurobiological bases of schizophrenic symptom clusters. Schizophr Bull 24:267-283

Olton DS, Papas BC (1979) Spatial memory and hippocampal function. Neuropsychologia 17:669-682

Olton DS, Wible CG, Shapiro ML (1986) Mnemonic theories of hippocampal function. Behav Neurosci 100:852-855

Ozawa K, Hashimoto K, Kishimoto T, Shimizu E, Ishikura H, Iyo M (2006) Immune activation during pregnancy in mice leads to dopaminergic hyperfunction and cognitive impairment in the offspring: a neurodevelopmental animal model of schizophrenia. Biol Psychiatry 59:546-554

Patterson PH (2002) Maternal infection: window on neuroimmune interactions in fetal brain development and mental illness. Curr Opin Neurobiol 12:115-118

Patterson PH (2007) Maternal effects on schizophrenia risk. Science 318:576-577

Patterson PH (2009) Immune involvement in schizophrenia and autism: etiology, pathology and animal models. Behav Brain Res 204:313-321

Pocivavsek A, Icenogle L, Levin ED (2006) Ventral hippocampal alpha7 and alpha4beta2 nicotinic receptor blockade and cloza- 
pine effects on memory in female rats. Psychopharmacology 188:597-604

Rahimi O, Claiborne BJ (2007) Morphological development and maturation of granule neuron dendrites in the rat dentate gyrus. Prog Brain Res 163:167-181

Reif A, Fritzen S, Finger M, Strobel A, Lauer M, Schmitt A, Lesch KP (2006) Neural stem cell proliferation is decreased in schizophrenia, but not in depression. Mol Psychiatry 11:514-522

Reif A, Schmitt A, Fritzen S, Lesch KP (2007) Neurogenesis and schizophrenia: dividing neurons in a divided mind? Eur Arch Psychiatry Clin Neurosci 257:290-299

Samuelsson AM, Jennische E, Hansson HA, Holmäng A (2006) Prenatal exposure to interleukin-6 results in inflammatory neurodegeneration in hippocampus with NMDA/GABA(A) dysregulation and impaired spatial learning. Am J Physiol Regul Integr Comp Physiol 290:R1345-R1356

Schmitt A, Weber S, Jatzko A, Braus DF, Henn FA (2004) Hippocampal volume and cell proliferation after acute and chronic clozapine or haloperidol treatment. J Neural Transm 111:91-100

Shapiro ML, Olton DS (1994) Hippocampal function and interference. In: Schacter DL, Tulving E (eds) Memory systems. MIT, London, pp 141-146

Sharma T, Hughes C, Soni W, Kumari V (2003) Cognitive effects of olanzapine and clozapine treatment in chronic schizophrenia. Psychopharmacology 169:398-403
Shi L, Fatemi SH, Sidwell RW, Patterson PH (2003) Maternal influenza infection causes marked behavioral and pharmacological changes in the offspring. J Neurosci 23:297-302

Steele RJ, Morris RG (1999) Delay-dependent impairment of a matching-to-place task with chronic and intrahippocampal infusion of the NMDA-antagonist D-AP5. Hippocampus 9:118-136

Tamminga CA (2006) The neurobiology of cognition in schizophrenia. J Clin Psychiatry 67(Suppl 9):9-13

Toro CT, Deakin JF (2007) Adult neurogenesis and schizophrenia: a window on abnormal early brain development? Schizophr Res 90:1-14

van Praag H, Schinder AF, Christie BR, Toni N, Palmer TD, Gage FH (2002) Functional neurogenesis in the adult hippocampus. Nature 415:1030-1034

Weinberger DR (1999) Cell biology of the hippocampal formation in schizophrenia. Biol Psychiatry 45:95-402

Zuckerman L, Weiner I (2005) Maternal immune activation leads to behavioral and pharmacological changes in the adult offspring. $\mathrm{J}$ Psychiatr Res 39:311-323

Zuckerman L, Rehavi M, Nachman R, Weiner I (2003) Immune activation during pregnancy in rats leads to a postpubertal emergence of disrupted latent inhibition, dopaminergic hyperfunction, and altered limbic morphology in the offspring: a novel neurodevelopmental model of schizophrenia. Neuropsychopharmacology 28:1778-1789 